\title{
Agricultura familiar e as teses de doutorado no Brasil
}

\author{
Doctoral thesis on familiar agriculture in Brazil
}

Elisângela dos Santos Faustino RÖDER'

Edna Lucia da SILVA²

\section{Resumo}

O artigo apresenta o mapeamento da produção científica em teses de doutorado sobre agricultura familiar produzidas no período de 2000 a 2009 nos programas de pós-graduação no Brasil. No Banco de Teses e Dissertações da Coordenação de Aperfeiçoamento de Pessoal de Nível Superior, foram encontradas 153 teses que possuíam como foco principal de abordagem a agricultura familiar. Os resultados das análises realizadas mostram que: a) o desenvolvimento de teses na temática teve crescimento gradual a partir de 2000, atingindo, no ano de 2009, 33 defesas; b) destacam-se como produtoras dessas teses as instituições localizadas nas Regiões Sul e Sudeste do Brasil; c) nessas 153 teses, foram identificadas 122 teses que tratavam de temas relacionados mais diretamente ao desenvolvimento rural e 21 à produção rural propriamente dita; e 10 teses, por serem abordagens únicas, foram agrupadas em uma categoria denominada outros; d) sobressaíram as abordagens a respeito das ações, das políticas e dos atores voltadas para o desenvolvimento rural, abrangendo 73 teses, nas quais estão incluídos trabalhos que trataram de ações educativas ( 16 teses); políticas públicas ( 13 teses), gestão (10 teses), assentamentos (7 teses), associativismo (7 teses), enfoques direcionados a atores específicos (6 teses), trabalho (6 teses), pluriatividade (5 teses) e legislação (3 teses). Conclui-se que o mapeamento realizado possibilitou inventariar a produção realizada no período analisado e mostrar indícios de que as abordagens são influenciadas pelo meio e pelo contexto, visto que os estados com maior número de produção em teses sobre a temática agricultura familiar são os que apresentam a atividade desse segmento da economia mais estruturada e desenvolvida.

Palavras-chave: Agricultura familiar. Comunicação científica. Teses.

\begin{abstract}
This article presents collected data from doctoral thesis about familiar agriculture defended at Brazilian graduate programs recognized from 2000 to 2009. The bank of thesis and dissertations of the Coordination for the Improvement of Higher Education Personnel was used to collect data. The corpus was composed of 153 doctoral theses whose main focus was on familiar agriculture. The results indicated that:a) the number of doctoral dissertations about familiar agriculture increased after 2000, totaling 33 doctoral theses in 2009; 6 ) the universities where the doctoral defenses occurred were mainly located in the Southern and Southeast regions of Brazil; c) 122 theses discussed rural development, 21 discussed rural production and the remaining ones discussed other specific topics; d) among the 73 dissertations that discussed rural development, 16 were related to education, 13 to public policies, 10 to management, 7 to settlement, 7 to rural associativism, 6 were devoted to specific interested parties, 6 discussed work, 5 pluriactivity, and 3 legislation. It may be concluded that the mapping enabled the collection of studies within the period analyzed and it showed evidence that these approaches are influenced by environment and context, since the states with a greater number of theses on familiar agriculture are those that have more economically structured and developed agricultural activities.
\end{abstract}

Keywords: Familiar agriculture. Scientific communication. Doctoral theses.

\footnotetext{
1 Mestre em Ciência da Informação. Florianópolis, SC, Brasil.

2 Professora Doutora, Universidade Federal de Santa Catarina, Programa de Pós-Graduação em Ciência da Informação. Campus Universitário Trindade, 88045-000, Florianópolis, SC, Brasil. Correspondência para/Correspondence to: E.L. SILVA. E-mail: <edna@cin.ufsc.br>.

Recebido em 13/9/2012 e aceito para publicação em 18/4/2013
} 


\section{Introdução}

As universidades brasileiras são o locus para o desenvolvimento da pesquisa científica em todas as áreas do conhecimento, e parte significativa do conhecimento científico é produzida nos programas de pós-graduação. A participação das universidades no desenvolvimento da sociedade acontece dessa maneira pelo fornecimento de resultados de pesquisa que possam auxiliar na inovação científico-tecnológica, na criação de novos processos e produtos, na solução de problemas, além de propiciar que se eleve o nível de qualificação de profissionais e pesquisadores (Velho, 2007).

Neste artigo, apresentam-se resultados de uma pesquisa cuja abordagem volta-se para os estudos da comunicação científica, especificamente para a produção científica em teses de doutorado com o foco de sua abordagem em agricultura familiar. Nessas teses, encontram-se pesquisas científicas sobre a agricultura familiar desenvolvidas em cursos de pós-graduação no Brasil. As teses de doutorado sobre agricultura familiar representam parte da contribuição efetiva dos programas de pós-graduação para esse setor da economia do País e representam o envolvimento dessas instituições com as temáticas de interesse da sociedade brasileira.

A produção científica na área da agricultura, no Brasil, teve início com a criação do Jardim Botânico e de alguns institutos de pesquisa distribuídos primeiramente nos estados da Bahia, de Pernambuco, de Sergipe, do Rio de Janeiro e do Rio Grande do Sul no século XIX. No começo do século XX, o Ministério da Agricultura implantou Estações Experimentais e essas colaboraram no processo de sistematização da produção científica desse setor da economia (Rodrigues, 1987).

No ano de 1972, com a criação da Empresa Brasileira de Pesquisa Agropecuária (Embrapa), a produção científica direcionada para a agropecuária ganhou uma forte aliada (Lima, 1993). A Embrapa, entre outros objetivos, estava para criar políticas de desenvolvimento científico-tecnológico, apoiar os pesquisadores, angariar recursos financeiros, programar, executar e avaliar os trabalhos de pesquisas (Rodrigues, 1987). Os cursos de pós-graduação em Ciências Agrárias também contribuíram com o crescimento significativo da produção científica a partir da década de 1970 (Lima, 1993).
Cabe ressaltar que a agricultura familiar, na última década no País, passou a ser vista pelo governo brasileiro como um segmento imprescindível para o combate à fome e para garantir segurança alimentar e nutricional. Estima-se que cerca de 70,0\% da alimentação do brasileiro provenha da agricultura familiar. O Estado, ciente dessa importância, passou a se preocupar em definir políticas públicas que atendessem às peculiaridades da agricultura familiar na tentativa de diversificar as formas de auxílio a essa categoria e fomentar a pluriatividade por meio de linhas de crédito específicas. Como parte desse processo, a aprovação da Lei no 11.947/2009 passou a garantir que "No mínimo 30,0\% do valor destinado por meio do Programa Nacional de Alimentação Escolar, do Fundo Nacional de Desenvolvimento da Educação, do Ministério da Educação", devia ser utilizado na aquisição da produção agrícola familiar (Brasil, 2009, p.1). O setor, segundo o Instituto Brasileiro de Geografia e Estatística (IBGE), é responsável pela empregabilidade de 74,4\% das ocupações no setor agrícola nacional, responsável por um terço da receita vinculada a essa produção, ocupando a fração de 24,3\% dos estabelecimentos agropecuários (Instituto Brasileiro de Geografia e Estatística, 2006).

Diante dessa conjuntura, três razões mobilizaram o desenvolvimento desta pesquisa: 1) a importância desse setor - agricultura familiar -, para a sociedade brasileira; 2) o fato de que mapeamentos da produção científica são importantes na medida em que mostram quem, onde, quando e o que foi produzido, permitindo inventariar e destacar o que foi privilegiado nas abordagens dos estudos e 3) a constatação de que estudos dessa natureza têm sido recorrentes na Ciência da Informação, visto que essa disciplina incorpora entre seus interesses os estudos dos processos de comunicação científica.

O desenvolvimento da pesquisa foi motivado pela obtenção de resposta para a seguinte questão: Quais as características gerais (quem, quando e onde) e temáticas principais (o quê) das teses produzidas a respeito da agricultura familiar nos programas de pós-graduação das universidades brasileiras? Na busca dessa resposta, foi definido como objetivo geral investigar as características das teses produzidas a respeito da agricultura familiar nos programas de pós-graduação das universidades brasileiras na última década (2000-2009). 


\section{A ciência, a Ciência da Informação e a comunicação científica}

Para contextualizar o desenvolvimento desta pesquisa na Ciência da Informação é preciso entender os processos de comunicação na ciência.

A ciência como um evento com data de referência, como um marco na história da humanidade, é, para Targino (2000, p.2), "Uma instituição social, dinâmica, contínua e cumulativa". Para facilitar a compreensão do que seja ciência é preciso entender o funcionamento do campo científico. O universo que compreende desde a produção até a disseminação da ciência e suas repercussões é denominado de campo científico por Bourdieu (2004).

A motivação do pesquisador nesse campo científico sustenta-se no desejo de aumentar seu capital científico, o que gera poder de duas espécies: poder institucional e institucionalizado e poder específico, prestígio pessoal. De acordo com Bourdieu (2004, p.26):

[...] o capital científico puro adquire-se, principalmente, pelas contribuições reconhecidas ao progresso da ciência, as invenções ou as descobertas (as publicações, especialmente nos órgãos mais seletivos e mais prestigiosos, [...]; o capital científico da instituição se adquire, essencialmente, por estratégias políticas (especificas) que têm em comum o fato de todas exigirem tempo - participação em comissões, bancas (de teses, de concursos), colóquios mais ou menos convencionais no plano científico, cerimônias, reuniões etc.

A produção científica produzida e legitimada entre os componentes do campo propicia ao cientista um capital que Ihe dá autoridade junto à comunidade científica, e essa the atribui poderes de participação na discussão sobre manter, alterar, legitimar e regularizar as leis que regem as relações entre ciência, cientista e sociedade (Bourdieu, 2004).

Consequentemente, já se tornou lugar-comum afirmar que o conhecimento só se corporifica e se consolida a partir de sua disseminação e legitimação por parte da comunidade científica. A disseminação de descobertas e de reflexões existe desde o momento em que o mundo da vida se tornou objeto de observação. Meadows (1999) relaciona a comunicação científica à tradição grega de forma oral, marcada pelas reuniões ao ar livre para discussões filosóficas, como as feitas por Aristóteles, e posteriormente pela forma escrita, também em registros gregos. A evolução da comunicação científica é proporcional à evolução da ciência em si. A cada nova descoberta surge a necessidade de comunicá-la, assim como a nova pesquisa apoia-se na análise de produções anteriores sobre o assunto. Para Bufrem et al. (2007) a publicação científica proporciona ao pesquisador a divulgação de seu trabalho como também promove e fortalece o ciclo de criação, organização e difusão do conhecimento.

A produção científica reflete os investimentos em Pesquisa \& Desenvolvimento realizados pelos países. Dantas (2004) lembra também que o Produto Interno Bruto (PIB) de países desenvolvidos tem vinculação com a sua produção científico-tecnológica. Na visão de Velho (2007), a produção científica de uma nação pode refletir suas demandas sociais.

Assim, a ciência como uma instituição socialmente constituída e mantida remete-se a um ambiente que reflete os anseios, os conflitos e as limitações de uma sociedade. Ela cresce na medida em que as forças internas de um campo se sobrepõem e se estendem para fora do campo científico. O importante é ressaltar que esse processo não é neutro, nem isento de interesses, sejam eles de influências do próprio campo, da comunidade científica, do ambiente político econômico ou social. Targino (2000) lembra que as características pessoais do pesquisador aparecem nos resultados das pesquisas, já que esse é um ser social, suas vivências e suas idiossincrasias se manifestam na sua produção científica.

Autores clássicos da área de informação já incorporaram os processos da comunicação científica como temática de interesse da Ciência da Informação. Nessa diretriz, incluem-se Mikhailov et al. (1984), que consideram a Ciência da Informação como uma disciplina que estuda a estrutura e as propriedades da comunicação científica nos quais se incluem os processos de comunicação nos canais formais e informais. Saracevic (1996, p.47), na mesma linha, concebe a Ciência da Informação como um “[...] campo dedicado às questões científicas e à prática profissional voltadas para os problemas de efetiva comunicação do conhecimento e de seus registros entre os seres humanos, no contexto social, institucional ou individual do uso e das necessidades de informação". 
A constituição da Ciência da Informação, como campo científico, possui algumas visões sobre a sua origem. Para Pinheiro (2002, p.72), os acontecimentos que propiciaram a constituição desse novo campo foram:

[...] o avanço científico e tecnológico, principalmente em função da 2a Guerra Mundial e, conseqüentemente, a explosão bibliográfica; a necessidade social, histórica, cultural e política do registro e transmissão dos conhecimentos e informações, produto do processo de desenvolvimento da Ciência e Tecnologia; e o surgimento de novas tecnologias a partir do microfilme e, principalmente, do computador.

A visão que atribui relação entre o surgimento da Ciência da Informação e o fenômeno da explosão da informação proveniente do renascimento científico ocorrido depois da 2a Guerra Mundial e, em decorrência, da necessidade de controle bibliográfico e de tratamento da documentação gerada nesse período é bem recorrente na literatura da área. Nessa perspectiva, segundo Miranda (2002), a sua origem esteve mais ligada à documentação do que propriamente à informação.

Para González de Gómez (2000, p.3), a Ciência da Informação surge no "Horizonte de transformações das sociedades contemporâneas que passaram a considerar o conhecimento, a comunicação, os sistemas de significado e os usos da linguagem como objetos de pesquisa científica e domínios de intervenção tecnológica". Como um novo campo, passou a incluir em seus "Programas de pesquisa e na definição do domínio de construção de seu objeto, traços e demandas da sociedade industrial". Dessa forma, passou-se a acompanhar "Os processos de reformulação dos paradigmas econômicos, sociais e políticos, desde a mercantilização da informação a sua reconfiguração como bem de capital e indicador de riqueza".

Realizada essa breve contextualização, deve-se acrescentar que a Ciência da Informação é uma ciência muito nova e sua existência é uma decorrência da institucionalização da ciência no mundo e das necessidades geradas por esse processo. A comunicação científica mostrou-se importante e passou a ser um sistema responsável pelo desenvolvimento da ciência. O processo de produção do conhecimento científico é expresso e mobilizado pelo processo de comunicação. Na definição clássica cunhada por Garvey (1979, p.ix), a comunicação científica incorpora "Todo espectro de atividades associadas com a produção, disseminação e uso da informação, desde o momento que o cientista gera sua ideia de pesquisa até o momento que os resultados de pesquisa são aceitos como componentes do conhecimento científico".

O ciclo da produção de conhecimento científico incorpora desde a produção, a publicação até a sua utilização por outros pesquisadores. A confiabilidade, uma das características da ciência, é obtida por duas vias, como ressalta Mueller (2000, p.22), o pesquisador deve utilizar uma rigorosa metodologia científica no desenvolvimento de sua pesquisa e submeter os resultados dessa para serem divulgados e submetidos ao julgamento de seus pares. Assim, a produção científica constituída pelo conjunto de publicações "Permite expor o trabalho dos pesquisadores ao julgamento constante de seus pares, em busca do consenso que confere a confiabilidade" ao conhecimento científico. Targino (2000, p.10), nessa mesma linha, acrescenta que a comunicação científica permite:

[...] somar os esforços individuais dos membros da comunidade científica. Eles trocam continuamente informações com seus pares, emitindo-as para seus sucessores e/ou adquirindo-as de seus predecessores. É a comunicação que favorece ao produto (produção científica) e aos produtores (pesquisadores), a necessária visibilidade e possível credibilidade no meio social em que o produto e produtores, se inserem.

A comunicação científica vai ao encontro da prerrogativa de que "Ciência é conhecimento público" (Ziman, 1984, p.84), ou seja, é um processo complexo que objetiva tornar público o que é produzido pelos cientistas, seja em forma de artigos, livros, teses e dissertações, que passam a ser representações das ideias, das descobertas, das inquietações e dos posicionamentos dos pesquisadores sobre um determinado tema. Por meio da análise da produção científica é possível identificar a construção e a reconstrução de um campo científico. Stumpf (2000) esclarece que a construção do conhecimento depende da comunicação, pois o avanço científico se apoia no conhecimento produzido para saber o que já é conhecido. Assim, a comunicação da ciência possibilita que se divulgue e se obtenha o que foi produzido e esse processo faz parte de um sistema amplo denominado comunicação científica. 
No Brasil, as pesquisas que abordam enfoques relacionados à temática da comunicação científica na Ciência da Informação foram iniciadas pelo Instituto Brasileiro de Bibliografia e Documentação (IBBD), hoje denominado Instituto Brasileiro de Informação em Ciência e Tecnologia (Ibict), órgão governamental responsável pela introdução desse campo de conhecimento no País com a implantação do primeiro mestrado em Ciência da Informação da América Latina, em 1970 (Couzinet et al., 2007; Silva etal., 2010).

Mundialmente, o século XX foi marcado pela intensificação da produção científica, gerando a necessidade de sistematização para torná-la visível para a sociedade. Para tanto, foram desenvolvidas tecnologias capazes de tornar o acesso e a distribuição da informação mais fácil e mais abrangente. Os veículos de informação se ampliaram, tornando o alcance menos complexo e mais amplo. A distância que separava os centros de pesquisa de professores ou outros interessados foi minimizada pela rede mundial de computadores, entre outras tecnologias.

No caso brasileiro, o aumento no número das pós- graduações, que são centros de excelência de produção científica, gerou forte crescimento qualiquantitativo na produção científica nacional. Segundo Dantas (2004), os agentes fomentadores e reguladores do Conselho Nacional de Desenvolvimento Científico e Tecnológico (CNPq), Coordenação de Aperfeiçoamento de Pessoal de Nível Superior (Capes), Financiadora de Estudos e Projetos (FINEP) etc. vêm investindo nos últimos anos em projetos mais específicos, ou seja, ligados de forma direta ao desenvolvimento socioeconômico do País. O intuito é proporcionar a alguns seguimentos ou setores da sociedade subsídios científicos específicos.

Apesar de todos os esforços empreendidos, a disseminação da produção científica ainda carece de instrumentos que possibilitem atingir 100\% de eficácia. $\mathrm{Na}$ universidade, apesar de todos os esforços para o controle e a disseminação da ciência produzida, ainda existe o que se denomina de literatura cinzenta. Das características da literatura cinzenta (grey literature) destaca-se o fato de que não é controlada por editores comerciais e não recebe numeração padronizada International Standard Serial Number (ISSN) e International Standard Book Number (ISBN). Assim, com reduzido número de exemplares, o seu acesso é dificultado pelos possíveis interessados em seu conteúdo (Gomes et al., 2000). Nos últimos anos, observaram-se várias tentativas para minimizar o problema de identificação e localização desse tipo de literatura facilitado pelas tecnologias, em especial pela Internet, que viabilizou a criação de repositórios institucionais e/ou especializados para se colocar disponível a produção técnica e científica de instituições e pessoas. No Brasil, desde a década de 1970, as universidades, a Capes e o Ibict têm realizado investimentos para a criação de repositórios de teses e dissertações, o que tem facilitado o acesso a esse tipo de literatura no formato digital.

As teses objetos de estudo desta pesquisa envolvem, conforme Campello (2000, p.121), uma "Revisão bibliográfica adequada, sistematização das informações existentes, planejamento e realização de trabalho necessariamente original". No Brasil, o termo tese está associado à obtenção do grau de doutor em instituições de ensino credenciadas pela Capes e elas são consideradas literatura cinzenta, pois "Não contam, na maioria dos casos, com um sistema de publicação e distribuição comercial". As teses são geralmente disponibilizadas em repositórios digitais e, nas Ciências Sociais e Humanidades, uma parte dessa produção geralmente transforma-se em livro. Meadows (1999, p.165) lembra que "As teses tem a extensão de um livro em termos de volume de conteúdo", mas para sua publicação como livro requerem modificações no estilo, na organização e nos pontos de destaque, o que implica quase sempre em reescrever toda a tese. Diante da realidade dessas áreas, boa parte do conteúdo dessa produção não é publicada em artigos, isso porque a condensação do conteúdo torna-se difícil. E não é publicada em livro porque o processo é muito trabalhoso, ficando então restrita sua divulgação aos meios digitais que ainda não foram totalmente incorporados como fonte de informação.

As colocações acima são importantes na medida em que, nesta pesquisa, a agricultura familiar foi analisada na perspectiva do olhar das teses de doutorado produzidas nos programas de pós-graduação do Brasil credenciados pela Capes. Pesquisas dessa natureza, com foco em outras temáticas, são frequentemente realizadas na área de Ciência da Informação; analisar dados de bases de produção científica desenvolvida nas universidades 
significa, conforme Kobashi e Santos (2006), criar meios para que se possa rememorar ou até reavaliar esse tipo de atividade nessas instituições. Para Kobashi (2007), o resgate dessas inscrições para analisá-las representa uma forma de promover a reapropriação desse conhecimento produzido.

\section{Métodos}

A metodologia de pesquisa aponta o caminho a ser percorrido na pesquisa proposta; apresenta meios capazes de organizá-la de forma a obter resultados confiáveis, sistemáticos, verificáveis; auxilia na administração dos custos, do tempo, dos contatos a serem feitos; enfim, possibilita a realização de um trabalho e o alcance de seus objetivos (Köche, 1997).

Do ponto de vista de seus objetivos, a pesquisa desenvolvida é caracterizada como exploratória e descritiva e, do ponto de vista da abordagem do problema, ela é qualiquantitativa. Quanto aos procedimentos técnicos adotados nesta pesquisa, de acordo com Gil (1991), caracterizam-se como pesquisa documental, porque seu corpus foi constituído de documentos, nesse caso teses de doutorado que ainda não receberam um tratamento analítico na perspectiva proposta nos objetivos desta pesquisa.

Nessa pesquisa, o foco da abordagem foi a produção científica oriunda dos cursos de doutorados de universidades brasileiras que abordam o assunto agricultura familiar, a qual foi produzida entre os anos $2000 \mathrm{e}$ 2009. O corpus da pesquisa foi constituído por teses cadastradas no Banco de Teses da Capes. A seleção do corpus foi realizada por meio da recuperação com o uso da palavra-chave agricultura familiar, selecionando "expressão exata", no campo denominado "assunto"e a leitura dos resumos para apurar essa seleção. As teses que constituíram o corpus da pesquisa estão indicadas no Anexo.

A análise dos dados foi realizada com base na não) que permitam a inferência de conhecimentos relativos às condições de produção/recepção (variáveis inferidas) destas mensagens.

As fases da análise de conteúdo são: 1) Pré-análise; 2) Exploração do material e 3) Tratamento dos resultados.

\section{Resultados}

Os resultados apresentados são provenientes da análise de 153 teses defendidas em diversos programas de pós-graduação do País. As teses que constituíram o corpus da pesquisa (Anexo) serão indicadas no texto pelo número recebido, ou seja,T1,T2 atéT153.

\section{Caracterização geral das teses sobre agricultura familiar}

O desenvolvimento de teses com a temática agricultura familiar no período de análise proposto nesta pesquisa mostra um crescimento gradual do número anual de produções que envolvem análises de diversos aspectos da produção da agricultura familiar. No ano de 2009, no qual se constataram 33 teses produzidas em diferentes programas de pós-graduação, percebeu-se que houve um aumento significativo de produção quando se compara com o ano de 2000 , no qual foram produzidas 7 teses nessa temática (Figura 1).

Quanto ao número de teses em relação à região de sua produção, as instituições localizadas nas Regiões Sul (Universidade Federal do Paraná (UFPR) - 18; Universidade Federal do Rio Grande do Sul (UFRGS) - 16; Universidade Federal de Santa Catarina (UFSC) - 15; Universidade Federal de Pelotas (UFPel) - 6; Universidade Estadual de Londrina (UEL) - 2; Universidade do Vale do Rio dos Sinos (Unisinos) - 1) e Sudeste (Universidade Estadual de Campinas (Unicamp) - 15; Universidade Estadual Paulista (Unesp) - 10; Universidade Federal de Viçosa (UFV) - 7; Universidade Federal Rural do Rio de Janeiro (UFRRJ) - 5; Universidade de São Paulo (USP) - 6; Pontifícia Universidade Católica de São Paulo (PUC-SP) - 4; Universidade Federal do Rio de Janeiro (UFRJ) - 2; Universidade Federal de São Carlos (UFSCar) - 2; Universidade Federal de Lavras (UFLA) - 1; Universidade Federal de Minas Gerais (UFMG) - 1) são responsáveis por $72,5 \%$ das produções de teses com o tema agricultura 


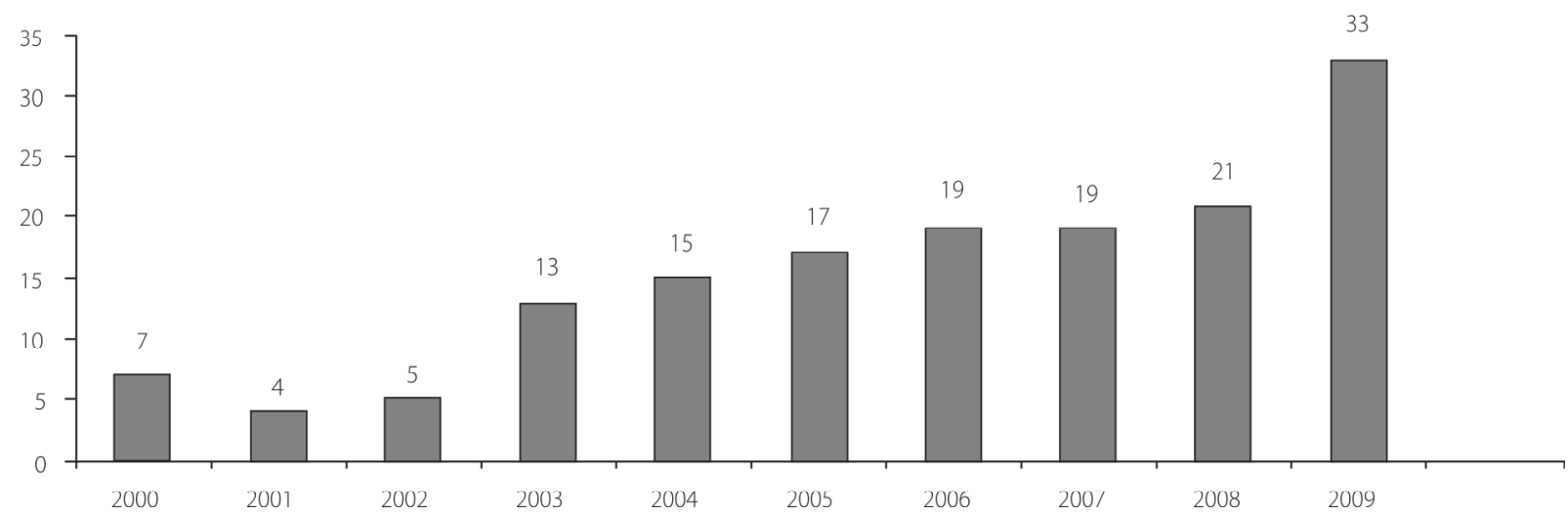

Figura 1. Teses sobre agricultura familiar (2000-2009). Distribuição por ano de defesa. Fonte: Dados da pesquisa levantados no Banco de Teses da Capes - 2010.

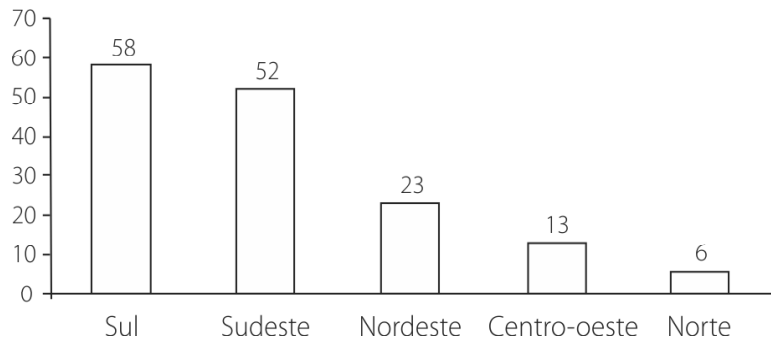

Figura 2. Teses sobre agricultura familiar (2000-2009). Distribuição da produção por região do Brasil.

Fonte: Dados da pesquisa levantados no Banco de Teses da Capes - 2010.

familiar no corpus desta pesquisa. Na sequência aparecem as instituições das Regiões Nordeste (Universidade Federal de Pernambuco (UFPE) - 9; Universidade Federal da Paraíba (UFPB) - 7; Universidade Federal de Campina Grande (UFCG) - 3; Universidade Federal do Ceará (UFC) - 1; Universidade Federal do Maranhã (UFMA) - 1; Universidade Federal do Rio Grande do Norte (UFRN) - 1; Universidade Federal do Sergipe (UFS) - 1), Centro-Oeste (Universidade de Brasília (UnB) - 11; Universidade Federal de Goiás (UFG) - 2) e Norte (Universidade Federal do Pará (UFPA) - 3; Universidade Federal Rural da Amazônia (UFRA) - 3) (Figura 2). A concentração da produção de teses nas Regiões Sul e Sudeste pode estar diretamente ligada à importância econômica dessa atividade na economia dessas regiões e ao interesse da comunidade científica a partir da implementação de políticas públicas para o setor. Para Guanziroli et al. (2001, p.120), "[...] por ser a principal área de concentração da agricultura familiar no Brasil, a
Região Sul é a que apresenta uma maior diversidade de sistemas e subsistemas produtivos". Ainda lembra que a Região Sudeste apresenta área relativamente menor ocupada por agricultores familiares em relação às Regiões Norte e Nordeste, mas sua participação no valor bruto da produção só perde para a Região Sul.

Para a classificação das áreas de conhecimento das teses da amostra desta pesquisa, foi consultada a classificação dos cursos no processo de avaliação da Capes em 2011. Os programas foram classificados considerando-se a grande área. Os programas que apresentaram o maior número de teses defendidas nos períodos analisados sobre o assunto na amostra desta pesquisa pertencem as seguintes grandes áreas: Multidisciplinar (41 teses), Ciências Humanas (40 teses), Ciências Agrárias (37 teses), Ciências Sociais Aplicadas (15 teses), Engenharias (9 teses), Educação (6 teses) (Figura 3) (Coordenação de Aperfeiçoamento de Pessoal de Nível Superior, 2011).

A diversidade das áreas, ou seja, 9 áreas, conforme classificação da Capes (Multidisciplinar, Ciências Humanas, Ciências Agrárias, Ciências Sociais Aplicadas, Engenharia, Educação, Ciências Exatas e da Terra, História e Ciências Sociais) e dos programas que abordaram a agricultura familiar nas teses de doutorado do corpus desta pesquisa, vêm reforçar que o tema tem amplo escopo e envolve muitos elementos para análise, podendo ser tratado a partir de diferentes enfoques, sejam de natureza econômica, social, educacional, política, agrárias, entre outros. As abordagens multidisciplinares são muito recorrentes nos estudos empreendidos sobre a temática, como 


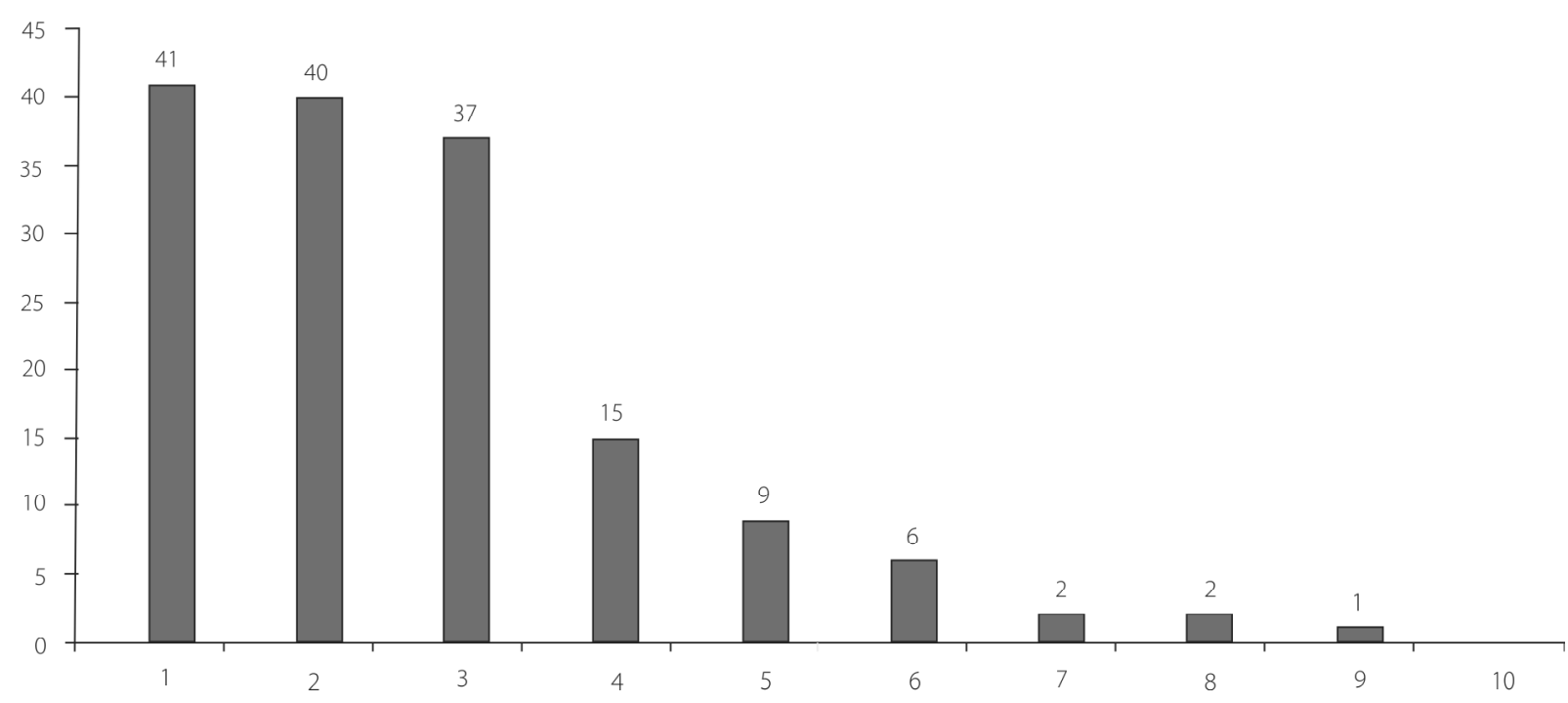

Figura 3. Teses sobre agricultura familiar (2000-2009). Distribuição por área dos programas de Pós-Graduação.

Fonte: Dados da pesquisa levantados no Banco de Teses da Capes - 2010.

mostram os resultados desta pesquisa, o que vem sustentar a constatação de que as possibilidades de análise do tema permitem que seja tratado por mais de uma perspectiva em uma mesma pesquisa.

\section{Análise de conteúdo dos resumos das teses sobre agricultura familiar}

Para a análise temática foram verificados os títulos, os resumos e as palavras-chave elaborados pelos autores das teses. Os resumos tiveram um papel importante para o seu desenvolvimento porque proporcionaram identificação da vinculação efetiva da tese com a temática agricultura familiar.

O processo de análise temática das teses do corpus desta pesquisa seguiu uma ordenação de dados, organizando-os em categorias, que segundo Bardin (1994), têm como objetivo fornecer por condensação uma representação simplificada dos dados brutos. O critério de categorização utilizado foi o semântico, que se processou com a reunião de temas com significados aproximados formando uma categoria temática. "Classificar elementos em categorias impõe a investigação do que cada um deles tem em comum com os outros. O que vai permitir o seu agrupamento é a parte comum existente entre eles" (Bardin, 1994, p.112). No caso desta pesquisa, os conteúdos das teses foram agrupados em categorias mais gerais, adotando-se uma perspectiva macro. Tais categorias foram desdobradas no momento da análise mais detalhadamente em subcategorias, adotando, sempre que possível, uma perspectiva cada vez menor para que se possibilitasse uma aproximação mais evidente com a temática da agricultura familiar. Tal procedimento foi adotado pelo fato do conteúdo de cada tese ser muito particular, visto a própria exigência de originalidade na abordagem dos temas desse tipo de trabalho científico nas universidades.

As teses com base em seu foco principal foram agrupadas em duas grandes temáticas: 122 teses referem-se mais diretamente ao desenvolvimento rural e 21 à produção rural propriamente dita. Abordagens únicas foram agrupadas na categoria denominada de outros. No caso de superposição de abordagens, adotou-se como critério a preponderância temática para a escolha da classificação.

\section{Desenvolvimento rural do ponto de vista da agricultura familiar}

O desenvolvimento rural em sentido amplo inclui estudos de impacto ambiental, ordenamento de território, monitoramento ambiental, planejamento, acompanhamento e avaliação do desenvolvimento socioeconômico desse espaço, bem como dos atores sociais envolvidos 
nas questões relacionadas a esse universo. O desenvolvimento rural incluirá dessa forma três eixos de análise: desenvolvimento rural com foco em ações, políticas e atores sociais (75 teses); desenvolvimento rural com foco em estudos de sustentabilidade (14 teses); e pesquisas direcionadas aos problemas regionais (33 teses).

\section{Desenvolvimento rural: ações, políticas e atores}

Nas teses analisadas nesta pesquisa, foram identificadas diversas abordagens demonstrando uma compreensão multifacetada do que representa o desenvolvimento rural com foco na agricultura familiar em termos de ações, políticas e atores. Nessa categoria, foram agrupadas pesquisas que tratavam de ações educativas (16 teses), políticas públicas (13 teses), gestão (10 teses), assentamentos (7 teses), associativismo (7 teses), pluriatividade (6 teses), enfoques direcionados a atores específicos (6 teses), trabalho (6 teses) e legislação (3 teses).

No Brasil, as ações e os programas de capacitação rural têm sido intensificados a partir do momento em que o setor produtivo da sociedade passou a ser dependente de conhecimento. Tais ações contribuem com o processo de desenvolvimento rural quando possibilitam ganhos nos aspectos relacionados tanto à produção quanto à conscientização sobre o melhor uso dos recursos naturais e a participação social. Em relação a essa subcategoria Ações educativas na agricultura familiarforam identificadas 16 teses. Verificou-se, ainda, que possuíam duas vertentes de abordagem, ou seja, 9 teses tratavam de ações educativas de escolas e projetos oficiais de educação rural (T1-T9) e 7 teses de ações educativas de extensão rural e assistência técnica rural (T10-T16).

As políticas públicas são instituídas para direcionar ações e recursos governamentais. Na subcategoria políticas públicas na agricultura familiar, foram identificadas 13 teses. Dessas, 7 abordam questões relacionadas ao Programa Nacional de Fortalecimento da Agricultura Familiar (PRONAF) (T17-T23). O PRONAF é um programa mantido pelo governo brasileiro que foi instituído em 1996 e tem passado por mudanças ao longo dos anos. 0 programa constitui-se como a primeira política pública direcionada para a agricultura familiar brasileira e visa financiar projetos individuais ou coletivos que gerem renda aos agricultores familiares e assentados da reforma agrária, com taxas de juros reduzidas se comparadas aos outros financiamentos rurais (Brasil, 2012). A concentração de estudos sobre esse programa deve-se ao fato de que especialistas dessa área, como Schneider et al. (2004), quando levantam o histórico e avaliam o PRONAF, constatam que essa política apesar de estar em constante processo de aperfeiçoamento ainda apresenta algumas lacunas. Contudo, admitem que esse, pelo curto período de sua vigência e de suas limitações, já apresentou significativa mudança no cenário nacional da agricultura. As seis demais são teses que adotaram perspectivas voltadas para questões institucionais e sociais referentes às políticas de desenvolvimento sustentável e territorial (T24-T29).

Os estudos voltados para a gestão da agricultura familiar sustentam-se na premissa de que técnicas gerenciais apropriadas para esse segmento contribuem para promover a sustentabilidade econômica dos empreendimentos. A gestão torna-se um importante processo na medida em que a agricultura familiar é um segmento que assume papel socioeconômico de grande importância no agronegócio brasileiro, além de contribuir para a inclusão social, razão pela qual o desenvolvimento desses empreendimentos é entendido como uma forma de tornar a sociedade mais justa social e economicamente. Nessa subcategoria Gestão na agricultura familiar, foram identificadas 10 teses voltadas para questões gerenciais relacionadas à agricultura familiar, incluindo desde as que criam modelos de gestão (T30-T31), as que avaliam as formas gerenciais e a eficiência da agricultura familiar (T32-T34), as que tratam da racionalidade administrativa nas propriedades (T35-T36), as que abordam a viabilidade econômica dos empreendimentos (T37-T38) até a que investiga o sistema de certificação de produtos orgânicos no Brasil (T39).

No Brasil, os assentamentos rurais são resultados da aplicação da política de reforma agrária e são considerados como estratégia para o desenvolvimento local. Nessa subcategoria que se refere aos assentamentos foram identificadas sete teses que abordam o processo de formação dos assentamentos (T40), as características dos assentamentos (T41), a sustentabilidade dos assentamentos (T42-T43), os sistemas produtivos dos assentamentos rurais (T44), a segurança alimentar (T45) e os índices de desempenho econômico de assentados da reforma agrária (T46). 
O associativismo na agricultura familiar é visto no País como uma forma de se criar meios e suportes para o fortalecimento da agricultura familiar e, em decorrência, passa a ser um indutor de desenvolvimento local. Nessa subcategoria associativismo na agricultura familiar estão incluídas sete teses que tratam de formas alternativas de organização da produção, do trabalho e da comercialização na agricultura familiar e que têm por base ações de cooperação do trabalho (T47-T53).

A agricultura familiar tem voltado sua atenção para a inclusão social das mulheres e dos jovens nessa atividade. O reconhecimento da importância das agricultoras tem chamado a atenção com o objetivo de torná-las economicamente ativas e protegidas pelas políticas governamentais. Quanto aos jovens, a preocupação principal é que estejam aptos e motivados para dar prosseguimento às atividades da família, garantindo a sobrevivência da atividade e, com isso, evitando-se o êxodo rural. Nessa subcategoria denominada atores na agricultura familiar foram identificadas seis teses que incluem abordagens sobre o papel, a vida e as conquistas das mulheres (T54-T57) e a situação dos jovens (T58-T59) na agricultura familiar.

Muitas questões estão envolvidas no trabalho na agricultura familiar, principalmente porque essa atividade tem uma forma social de trabalho e produção que se articula em torno de um grupo doméstico e seu modo específico de organizar e executar os processos de produção. A lógica que permeia essa organização passa pela ideia de aproveitamento máximo da força de trabalho dos membros da própria família, inclusive das crianças e dos jovens, como forma de garantir a viabilidade econômica dos empreendimentos. Dessa forma, o trabalho infanto-juvenil infelizmente faz parte da realidade dos empreendimentos de agricultura familiar no Brasil e, por isso, é um tema recorrente nas teses analisadas. Nessa subcategoria Trabalho na agricultura familiarforam identificadas seis teses que abordam questões ligadas ao trabalho no campo, incluindo exploração do trabalho (T60-T62), novas tendências na organização do trabalho (T63-T64) e saúde mental do trabalhador da agricultura familiar (T65).

Pluriantividade refere-se à diversificação das atividades e à ampliação de fontes alternativas de renda na agricultura familiar. As cinco teses classificadas nessa subcategoria Pluriatividade na agricultura familiar abordam os fatores que levam os produtores a direcionarem suas estratégias para o aumento de renda fora da unidade produtiva e a mudança na organização do trabalho familiar no que tange às atividades econômicas e produtivas (T66-T70).

A agricultura familiar no Brasil percorreu um longo caminho marcado por lutas sociais e hoje é uma atividade institucionalizada pelas políticas públicas de desenvolvimento rural. A legislação que regula a atividade no País faz parte dessas conquistas. Na subcategoria Legislaçãoe a agricultura familiar foram identificadas três teses que focalizam os impactos da Legislação sobre a produção familiar na agricultura (T71-T73).

\section{Desenvolvimento rural: estudos regionais}

As 35 teses, incluída subcategoria estudos regionais, caracterizam-se por análises que abordam questões relacionadas aos aspectos ambientais e à produção agrícola do tipo familiar. Entre esses aspectos estão: formas de ocupação do território e como esse processo influencia na conduta do agricultor e nas relações com o meio; produções específicas da região, envolvendo principalmente a análise de práticas produtivas quanto à sustentabilidade e a eficácia econômica e caracterização; particularidades regionais de relacionamento com o meio ambiente e com a sociedade; projetos que almejam desenvolvimento regional; e ações de atores sociais. Tais estudos regionais foram descritos a seguir concentrando-os nos estados da federação brasileira

Quanto aos estudos regionais referentes ao $\mathrm{Pa}$ raná, percebe-se, dentre os nove estudos, uma concentração de cinco análises na Região Metropolitana de Curitiba (T74-T78). Outros três estudos dessa subcategoria abordam aspectos mais amplos e relacionados ao estado do Paraná (T79-T81). Apenas um deles apresenta um estudo mais pontual, analisando a Região da Itapejara do Oeste e focalizando as transformações decorrentes da diversidade do "meio de vida" no meio rural (T82). Os estudos referentes a São Paulo tiveram enfoques bem pontuais nas sete pesquisas em que foram objetos de estudos a Microrregião de Presidente Prudente (T83), o Vale do Ribeira (T84), o município de São José do Rio Preto (T85), os municípios de Bilac, Birigui e Guararapes 
(T86), os bairros rurais em Anhumas (T87), o Escritório de Desenvolvimento Rural do Município de Jales (T88), os produtores de laranja do município de Bebedouro (T89). Dos cinco estudos identificados referentes à Santa Catarina, três deles abordam o Oeste catarinense (T90-T92). Os dois outros estudos trataram de aspectos diversos, sendo um deles sobre o processo de conservação dos remanescentes da Mata Atlântica em Santa Catarina (T93) e o outro sobre a atuação das companhias colonizadoras no início do século XX e seu papel na reconstrução do município de Cruzeiro, atual Joaçaba (T94). O estado de Pernambuco foi focalizado por três pesquisas, duas delas trataram de estudos sobre irrigação nos município de Pesqueira (T95), Petrolândia e Floresta (T96) e o terceiro tratou da política de desenvolvimento territorial e da capacidade de adaptação da agricultura familiar no submédio (T97). A região da Amazônia também foi contemplada com três teses: a primeira tratou dos modelos de paisagens na ocupação da Rodovia Transamazônica (T98); a segunda sobre a prevenção aos incêndios florestais na Amazônia (T99); e a terceira a respeito das dinâmicas das relações sociedade-natureza em área de fronteira agrária na Amazônia oriental (T100). Em relação ao estado do Rio Grande do Sul, foram identificados dois estudos, um deles focaliza uma comunidade do município de Pelotas (T101) e outro, mais abrangente, o próprio estado (T102). Ainda cabe registrar que os estados do Ceará (T103), da Paraíba (T104), do Piauí (T105), do Rio Grande do Norte (T106) e de Roraima (T107) foram contemplados com um estudo cada um. A região do II Departamento de San Pedro no Paraguai foi contemplada com um estudo (T108), o único estudo direcionado a analisar essa questão em outro país.

\section{Desenvolvimento rural: sustentabilidade}

Nesta categoria foram incluídas 14 teses que abrangem ações que visam avaliar, descrever e propor melhoria no uso dos recursos naturais. É perceptível nesses trabalhos a preocupação com a sustentabilidade do ponto de vista ambiental e econômico. Geralmente, entende-se como desenvolvimento sustentável aquele que alia o desenvolvimento social ao progresso econômico eà manutenção e à conservação dos recursos naturais, visando diminuir os impactos econômicos sobre o meio ambiente. Optou-se, na apresentação dessa categoria, pela divisão das teses que tratavam da sustentabilidade do ponto de vista da agroecologia, dos indicadores de sustentabilidade e das práticas agrícolas preservacionistas.

A agroecologia refere-se ao manejo ecologicamente responsável dos recursos naturais. No desenvolvimento rural sustentável com relação à agroecologia foram identificadas 10 teses que avaliaram, caracterizaram, investigaram a adoção, as práticas ecológicas adotadas na agricultura familiar e, em especial, a organização da produção de orgânicos. As teses concentram estudos realizados nos últimos anos da década analisada nesta pesquisa e trataram mais especificamente de duas vertentes, ou seja, de analises de movimento agroecológico no âmbito da agricultura familiar (T109-T115) e de práticas agroecológicas evidenciando a produção de orgânicos (T116-T118).

Quanto aos indicadores no desenvolvimento rural sustentável, duas teses propõem a construção de indicadores que possibilitariam avaliar o grau de sustentabilidade das práticas e dos manejos adotados pela agricultura familiar (T119-T129).

As teses que abordaram as práticas agrícolas no desenvolvimento rural sustentável foram duas, uma que estuda as práticas dos Índios Terena e dos caboclos da região do Pantanal (T121) e outra que estuda as práticas agrícolas de agricultores com o objetivo de identificar os fatores que interferem nas escolhas realizadas pelos agricultores (T122).

\section{Produção rural do ponto de vista da agricultura familiar}

A produção agrícola familiar brasileira está baseada no uso da mão de obra dos membros que compõem a unidade produtiva. Tal particularidade reflete em uma estrutura diferenciada de relações sociais internas e externas ao grupo, em uma racionalidade distinta na forma administrativa e na relação com os meios de produção, o tipo de cultivo, entre outras.

Essa subcategoria teve 21 teses analisadas, que foram divididas em estudos do solo, culturas, recursos hídricos.

Nessa subcategoria da produção rural, oito teses contemplaram os estudos do solo. O solo é uma questão 
importante na agricultura familiar, pois a sua composição ou o seu manejo contribuem para o resultado e a continuidade da produção. As análises realizadas revelaram características dos solos, formas de manejo do ponto de vista da sustentabilidade, a necessidade de mudança de prática com o uso de produtos químicos e/ou adubo orgânico, avaliação das formas de utilização do solo, entre outros aspectos. Identificam-se nesses estudos cinco teses que avaliam a composição do solo (T123-127) e três que avaliam as formas de manejo (T128-T130).

Nessa subcategoria da produção rural, oito teses contemplaram as culturas. Nessas encontram-se abordagens sobre várias culturas, tais como cítricos (T131); hortaliças (T132); pequi (T133); oleaginosas (T134-T137) e algodão (T138). Tais estudos avaliaram a produção, as formas de comercialização e as condições climáticas em relação à eficácia econômica para propor melhorias no cultivo de determinados plantios.

Na subcategoria produção rural com referência aos recursos hídricos como importante fator produtivo, a água também se tornou objeto de análise como recurso natural esgotável. Em cinco teses foram avaliadas as formas de uso dos recursos hídricos pelos agricultores, ou a implantação de culturas irrigadas (T139-T142) e as medidas preservacionistas como controle da poluição dos recursos hídricos (T143).

\section{Outros}

Esta categoria compreende 10 teses que se caracterizaram por estudos que trataram de temas cujo foco era a agricultura familiar, contudo não se enquadravam nas categorias e subcategorias acima colocadas. Os temas tratados foram: inclusão digital na agricultura familiar (T144); desenvolvimento e avaliação de um descaroçador de algodão (T145); o processo social de sucessão da propriedade na agricultura familiar (T146); modelo de análise do meio físico, tendo como base a geologia, a fim de identificar os aspectos geoquímicos num mapeamento de unidades de paisagem (T147); direitos sociais dos agricultores familiares, especificamente relacionados à previdência social (T148); projeto de tratamento do esgoto doméstico para a agricultura familiar (T149); vulnerabilidade dos agricultores familiares quanto ao uso de agrotóxicos (T150); qualidade de vida da agricultura familiar brasileira (T151); estágio de modernização de uma unidade agrícola (T152); e mudanças na vida dos agricultores familiares no que tange à sua reprodução social, à qualidade de vida no campo, à utilização e a influência das tecnologias usadas na produção agropecuária e à visão da sociologia diante dessas transformações (T153).

\section{Considerações Finais}

O objetivo desta pesquisa foi inventariar a produção científica sobre o tema agricultura familiar, focalizando os relatórios produzidos pelos doutorandos de cursos de pós-graduação de universidades recomendados pela Capes. O recorte da pesquisa compreendeu as teses produzidas entre os anos 2000 e 2009. Nesse movimento de mapeamento, identificou-se um conjunto de regularidades sugerindo algumas características para as teses com o foco principal em agricultura familiar desenvolvidas nos programas de pós-graduação, que podem ser resumidos da seguinte forma, em relação:

- à região das defesas e às instituições que se destacaram e estão localizadas nas Regiões Sul (UFPR, UFRGS, UFSC, UFPEL, UEL, Unisinos) e Sudeste (Unicamp, Unesp, UFV, USP, UFRRJ, PUC-SP, UFRJ, UFSCar, UFLA, UFMG) e são responsáveis por 72,5\% do total das teses.

- às grandes áreas do conhecimento dos programas, conforme classificação da Capes, que se destacaram na pesquisa da temática agricultura familiar são: Multidisciplinar (41 teses), Ciências Humanas (40 teses), Ciências Agrárias (37 teses), Ciências Sociais Aplicadas (15 teses), Engenharias ( 9 teses), Educação (6 teses), ressaltando-se que a temática foi abordada em 9 grandes áreas no total, tomando como referência novamente a classificação de áreas da Capes.

- à análise temática, 122 teses que tratam de temas relacionados mais diretamente ao desenvolvimento rural e 21 à produção rural propriamente dita. Às temáticas únicas, 10 teses foram agrupadas em uma categoria denominada de outros.

- ao cômputo geral sobressaíram-se as abordagens cujo enfoque principal foi dirigido para ações, políticas e atores como abordagens voltadas para o desenvolvimento rural, abrangendo 73 teses nas quais estão incluídas as que trataram de ações educativas (16 teses); políticas 
públicas (13 teses), gestão (10 teses), assentamento (7 teses), associativismo (7 teses), enfoques direcionados a atores específicos (6 teses), trabalho (6 teses), pluriatividade (5 teses) e legislação (3 teses).

O mapeamento realizado possibilitou inventariar a produção no período analisado e mostrar indícios de que as abordagens são influenciadas pelo meio e contexto visto de que os estados com maior número de produção em teses sobre a temática agricultura familiar são os que apresentam atividades econômicas e sociais mais estruturadas e desenvolvidas com relação à agricultura familiar.

Os dados analisados permitam inferir que a agricultura familiar é um setor que apresenta relações estreitas com o Estado, principalmente no âmbito de políticas públicas. Essa relação se expressa nos investimentos direcionados à estrutura de produção e reprodução do setor e na normatização e execução de projetos que viabilizam o cumprimento das funções sociais, econômicas e ambientais designadas à agricultura familiar que aparecem subjacentes nas abordagens das teses analisadas.

O mapeamento realizado possibilitou a localização e a classificação das pesquisas desenvolvidas sobre

\section{Referências}

BARDIN, L. Análise do conteúdo. Lisboa: Edições 79, 1994.

BOURDIEU, P. Os usos sociais da ciência: por uma sociologia clínica do campo científico. São Paulo: Unesp, 2004.

BRASIL. Fundo Nacional de Desenvolvimento da Educação. O encontro da agricultura familiar com a alimentação escolar: cartilha. Brasília: FNDE, 2009.

BRASIL. Ministério do Desenvolvimento Agrário. Programa nacional de fortalecimento da agricultura. Brasília: MDA, 2012. Disponível em: <http://portal.mda.gov.br/portal/saf/ programas/pronaf>. Acesso em: 12 maio 2012.

BUFREM, L.S. et al. Produção científica em ciência da informação: análise temática em artigos de revistas brasileira. Perspectivas em Ciência da Informação, v.12, n.1, p.38-49, 2007.

CAMPELLO, B.S. Teses e dissertações. In: CAMPELLO, B.S.; CENDÓN, B.V.; KREMER, J.M. (Org.). Fontes de informação para pesquisadores e profissionais. Belo Horizonte: UFMG, 2000. p.121-128.

COORDENAÇÃO DE APERFEIÇOAMENTO DE PESSOAL DE NIVEL SUPERIOR. Cursos recomendados ereconhecidos. Brasília: Capes, 2011. Disponível em: <www.capes.gov.br/avaliacao/ cursos-recomendados-e-reconhecidos>. Acesso em: 11 nov. 2011 a agricultura familiar nos programas de pós-graduação das universidades brasileiras permitindo evidenciar o comprometimento dos programas com as abordagens desse tema de interesse da sociedade. As teses, como componente da literatura científica, são representações do pensamento e das inquietações dos pesquisadores e da ciência desenvolvida no País. A agricultura familiar como um segmento importante da economia nacional tem recebido atenção das universidades revelada nesta pesquisa pela diversidade de estudos e pelo número de programas de pós-graduação envolvidos com a temática, o que vem demonstrar que tais instituições nacionais têm interesse nesse importante setor da economia do País, o que também mostra sintonia das universidades com a nova agenda de desenvolvimento nacional, na qual se tem fortes indícios de que agricultura familiar tem participação assegurada.

Espera-se que a análise empreendida possa compor um panorama dos estudos desenvolvidos nos programas de pós-graduação do Brasil sobre a agricultura familiar como representação do interesse e envolvimento das instituições de ensino superior com os problemas nacionais.
COUZINET, V.; SILVA, E.L.; MENEZES, E.M. A ciência da informação na França e no Brasil. Datagramazero: Revista de Ciência da Informação, v.8, n.6, 2007. Disponível em: <www. dgz.org.br>.

DANTAS, F. Responsabilidade social e pós-graduação no Brasil: idéias para (avali)ação. Revista Brasileira de Pós-Graduação, v.1, n.2, p.160-172, 2004

GARVEY,W.D. Communication: the essence of science. Oxford: Pergamon, 1979.

GIL, A.C. Como elaborar projetos de pesquisa. 3.ed. São Paulo: Atlas, 1991.

GOMES, S.L.R.; MENDONÇA, M.A.R.; SOUZA, C.M. Literatura cinzenta. In: CAMPELLO, B.S.; CENDÓN, B.V.; KREMER, J.M. (Org.). Fontes de informação para pesquisadores e profissionais. Belo Horizonte: UFMG, 2000. p.97-110.

GONZÁLEZ DE GÓMEZ, M.N. Metodologia de pesquisa no campo da ciência da informação. Datagramazero: Revista de Ciência da Informação, v.1, n.6, 2000. Disponível em: <www. dgz.org.br>.

GUANZIROLI, C. et al. Agricultura familiar e reforma agrária no século XXI. Rio de Janeiro: Garamond, 2001.

INSTITUTO BRASILEIRO DE GEOGRAFIA E ESTATÍSTICA. Agricultura familiar. Rio de Janeiro: IBGE, 2006. Disponível em: 
<http://www.ibge.gov.br/home/presidencia/noticias/ noticia_visualiza.php?id_noticia=1466\&id_pagina $=1>$. Acesso em: 5 jul. 2011.

KOBASHI, N.Y. Estudos de institucionalização social e cognitiva da pesquisa científica no Brasil: reflexões sobre um programa de pesquisa. In: LARA, M.L.G.; FUJINO, A.; NORONHA, D.P. (Org.). Informação e contemporaneidade: perspectivas. Recife: Néctar, 2007. p.185-197.

KOBASHI, N.Y.; SANTOS, R.N.M. Arqueologia do trabalho imaterial: uma aplicação bibliométrica à análise de dissertações e teses. In: ENCONTRO NACIONAL DE PESQUISA CIÊNCIA DA INFORMAÇÃO, 7., 2006, Marília. Anais... Marília: Unesp, 2006. p.1-15.

$\mathrm{KÖCHE}$, J.C. Fundamentos de metodologia científica: teoria da ciência e iniciação à pesquisa. Petrópolis: Vozes, 1997.

LIMA, M.F.B.F. Produção científica: revisão parcial da literatura brasileira com ênfase na área da agricultura. Ciência da Informação, v.22, n.3, p.233-236, 1993.

MEADOWS, A.J. A comunicação científica. Brasília: Briquet de Lemos, 1999.

MIKHAILOV, A.I.; CHERNYI, A.I.; GILIAREVSKII, R.S. Scientific communications and informatics. Arlington: Information Resources, 1984

MIRANDA, A. A ciência da informação e a teoria do conhecimento objetivo: um relacionamento necessário. In: AQUINO, M.A. O campo da ciência da informação: gênese, conexões e especificidade. João Pessoa: UFPB, 2002. p.9-24.

MUELLER, S.P.M. A ciência, o sistema de comunicação científica e a literatura científica. In: CAMPELLO, B.S.; CENDÓN, B.V.; KREMER, J.M. (Org.). Fontes de informação para pesquisadorese profissionais. Belo Horizonte: UFMG, 2000. p.21-34.
PINHEIRO, L.V.R. Gênese da ciência da informação ou sinais anunciadores da nova área. In: AQUINO, M.A. O campo da ciência da informação: gênese, conexões e especificidades. João Pessoa: UFPB, 2002. p.61-86.

RODRIGUES, C.M. Gênese e evolução da pesquisa agropecuária no Brasil: da instalação da corte portuguesa ao início da república. Caderno de Difusão de Tecnologia, v.4, n.1, p.21-38, 1987.

SARACEVIC, T. Ciência da informação: origem, evolução e relações. Perspectivas em Ciência da Informação, v.1, n.1, p.41-62, 1996.

SCHNEIDER, S.; CAZZELA, A.A.; MATTEI, L. Histórico, caracterização e dinâmica recente do PRONAF - Programa de Fortalecimento da Agricultura Familiar. In: SCHNEIDER, S.; SILVA, M.K.; MARQUES, P.E.M. (Org.). Políticas públicas e participação social no Brasil rural. Porto Alegre: UFRGS, 2004. p.21-49.

SILVA, E.L.; TAVARES, A.L.L.; PEREIRA, J.P.S. O estado da arte da pesquisa sobre comunicação científica (1996-2006) realizada no Brasil no âmbito da ciência da informação. Transinformação, v.22, n.3, p.207-223, 2010.

STUMPF, I.R. A comunicação da ciência na universidade: o caso da UFRGS. In: MUELLER, S.; PASSOS, E. (Org.). Comunicação científica. Brasília: UnB, 2000. p.107-121.

TARGINO, M.G. Comunicação científica: uma revisão de seus elementos básicos. Informação \& Sociedade, v.10, n.2, p.67-85, 2000.

VELHO, L. O papel da formação de pesquisadores no sistema inovação. Ciência e Cultura, v.59, n.4, p.23-28, 2007.

ZIMAN, J. Conhecimento público. São Paulo: USP, 1984 
Anexo

Corpus da pesquisa

\begin{tabular}{|c|c|c|c|c|c|c|c|}
\hline No & Doutores & Universidade & Defesa & No & Doutores & Universidade & Defesa \\
\hline $\mathrm{T} 1$ & Queiroz, J.B.P. & UnB & 2004 & $\mathrm{~T} 78$ & Hora, K.E.R. & UFPR & 2009 \\
\hline $\mathrm{T} 2$ & Oliveira, E.R. & UFLA & 2005 & $\mathrm{~T} 79$ & Nascimento, H.M. & Unicamp & 2005 \\
\hline $\mathrm{T} 3$ & Dansa, C.V.A. & UnB & 2008 & T80 & Poubel, M.B.P. & UFRRJ & 2005 \\
\hline T4 & Oliveira, M.A. & UFPR & 2008 & T81 & Lionço, V. & UFPel & 2007 \\
\hline T5 & Ramos, C.E.P. & UFPR & 2008 & T82 & Perondi, M.Â. & UFRGS & 2007 \\
\hline T6 & Dias, M.C.S. & UFMG & 2009 & T83 & Hespanhol, R.A. & Unesp & 2000 \\
\hline T7 & Estevam, D.O. & UFSC & 2009 & T84 & Abreu, I.S. & UFCG & 2002 \\
\hline T8 & Freire, J.C.S. & UFPA & 2009 & T85 & Sant'ana, A.L. & Unesp & 2003 \\
\hline T9 & Nogueira, L.C.A. & Unicamp & 2000 & T86 & Santo, C.R.E. & Unesp & 2005 \\
\hline $\mathrm{T} 10$ & Xavier, O.S. & PUC-SP & 2000 & T87 & Oliveira, E. & UnB & 2006 \\
\hline T11 & Pereira, E.Q. & UFPB & 2004 & T88 & Petinari, R.A. & Unicamp & 2007 \\
\hline $\mathrm{T} 12$ & Pinto, L.B. & Unicamp & 2005 & T89 & Chalita, M.A.N. & UFRGS & 2004 \\
\hline $\mathrm{T} 13$ & Sobral, F.J.M. & Unicamp & 2005 & T90 & Flexor, G.G. & UFRRJ & 2002 \\
\hline T14 & Ieno Neto, G. & UFPB & 2005 & T91 & Mior, L.C. & UFSC & 2003 \\
\hline T15 & Gritti, S.M. & UFRGS & 2007 & T92 & Mello, M.A. & UFRGS & 2009 \\
\hline T16 & Macedo, R.B. & UFPR & 2008 & T93 & Dalmora, E. & UFSC & 2004 \\
\hline $\mathrm{T} 17$ & Matos Filho, J. & Unicamp & 2002 & T94 & Radin, J.C. & UFSC & 2006 \\
\hline T18 & Altafin, I.G. & UnB & 2003 & T95 & Monteiro, A.L.N. & UFPE & 2007 \\
\hline T19 & Melo, L.A. & UFPE & 2003 & T96 & Carvalho, R.O. & UFPE & 2009 \\
\hline $\mathrm{T} 20$ & Brasil, I.C.P. & UnB & 2004 & T97 & Barros, E.R. & UFSC & 2007 \\
\hline T21 & Lima, V.L.C. & UFPE & 2006 & T98 & Venturieri, A. & Unesp & 2003 \\
\hline $\mathrm{T} 22$ & Hillig, C. & UFRGS & 2008 & T99 & Costa, L.M. & UFPA & 2004 \\
\hline $\mathrm{T} 23$ & Rocha, F.E.C. & UFPB & 2008 & $\mathrm{~T} 100$ & Oliveira, M.C.C. & UFRGS & 2009 \\
\hline T24 & Medeiros, C.M.V. & USP & 2003 & T101 & Salamoni, G. & Unesp & 2000 \\
\hline $\mathrm{T} 25$ & Costa, F.B. & UFRN & 2005 & T102 & Conterato, M.A. & UFRGS & 2008 \\
\hline T26 & Souza, C.R. & UFPR & 2006 & T103 & Holanda, F.U.X. & UFC & 2003 \\
\hline $\mathrm{T} 27$ & Alves, A.F. & UFSC & 2008 & $\mathrm{~T} 104$ & Ramos, N.L. & UFPB & 2009 \\
\hline $\mathrm{T} 28$ & Costa, R.C. & USP & 2008 & $\mathrm{~T} 105$ & Pereira, F.C. & UFRGS & 2003 \\
\hline $\mathrm{T} 29$ & Alves, A.F. & UFCG & 2009 & T106 & Nunes, E.M.O & UFRGS & 2009 \\
\hline $\mathrm{T} 30$ & Lourenzani, W.L. & UFSCar & 2005 & $\mathrm{~T} 107$ & Sousa Junior, A.F. & UnB & 2006 \\
\hline T31 & Sampaio, C.A. & UFRA & 2008 & $\mathrm{~T} 108$ & Figueiredo, O.A.T & UFRGS & 2008 \\
\hline $\mathrm{T} 32$ & Nunes, R. & USP & 2000 & T109 & Mariano Neto, B. & UFPB & 2006 \\
\hline $\mathrm{T} 33$ & Pereira Filho, C.A. & USP & 2001 & $\mathrm{~T} 110$ & Luzzi, N. & UFRRJ & 2007 \\
\hline $\mathrm{T} 34$ & Almeida, L. & UFPR & 2003 & $\mathrm{~T} 111$ & Gonçalves, S. & Unesp & 2008 \\
\hline $\mathrm{T} 35$ & Oliveira, J.T.A. & Unicamp & 2000 & $\mathrm{~T} 112$ & Verona, L.A.F. & UFPel & 2008 \\
\hline $\mathrm{T} 36$ & Roesler, D.A. & UFPR & 2009 & $\mathrm{~T} 113$ & Antônio Neto, F. & UFCG & 2009 \\
\hline $\mathrm{T} 37$ & Fernandes, A.R. & UFV & 2004 & $\mathrm{~T} 114$ & Batistela, E.M. & UFPR & 2009 \\
\hline T38 & Santos, M.J.C. & USP & 2005 & $\mathrm{~T} 115$ & Rodrigues, M.C.A. & UFRGS & 2009 \\
\hline T39 & Medaets, J.P.P. & UnB & 2003 & $\mathrm{~T} 116$ & Schmitt, C.J. & UFRGS & 2001 \\
\hline $\mathrm{T} 40$ & David, C. & UFSC & 2005 & $\mathrm{~T} 117$ & Ruszczyk, J.C. & UFPR & 2007 \\
\hline $\mathrm{T} 41$ & Anjos, M.B. & UFRRJ & 2003 & $\mathrm{~T} 118$ & Nobre Júnior, A. & UFRRJ & 2009 \\
\hline $\mathrm{T} 42$ & Silva, M.A.D. & UFG & 2006 & $\mathrm{~T} 119$ & Azevedo, R.A.B. & UFV & 2001 \\
\hline $\mathrm{T} 43$ & Silva, L.G.T. & UFPA & 2007 & $\mathrm{~T} 120$ & Tavares, E.D. & UnB & 2004 \\
\hline T44 & Rosa, L.A.B. & UEL & 2009 & $\mathrm{~T} 121$ & Silva, M.J. & USP & 2004 \\
\hline $\mathrm{T} 45$ & Silva, J.R.S. & UFMA & 2007 & $\mathrm{~T} 122$ & Sandrini, G.B.D. & UFPE & 2009 \\
\hline $\mathrm{T} 46$ & Silva Junior, R.P. & UFG & 2003 & $\mathrm{~T} 123$ & Oliveira, P.C. & UFRA & 2005 \\
\hline $\mathrm{T} 47$ & Olivo, C.J. & UFSC & 2001 & $\mathrm{~T} 124$ & Pereira, C.M. & Unicamp & 2007 \\
\hline $\mathrm{T} 48$ & Karam, K.F.F. & UFPR & 2003 & $\mathrm{~T} 125$ & Sawaki, H.K. & UFRA & 2007 \\
\hline $\mathrm{T} 49$ & Badalotti, R.M. & UFSC & 2004 & $\mathrm{~T} 126$ & Galvão, S.R.S. & UFPE & 2008 \\
\hline T50 & Cabral, L.O. & UFSC & 2004 & $\mathrm{~T} 127$ & Xavier, F.A. & UFV & 2009 \\
\hline T51 & Prim, L.F. & PUC-SP & 2004 & $\mathrm{~T} 128$ & Lanillo, R.F. & UEL & 2007 \\
\hline $\mathrm{T} 52$ & Schröder, M. & Unicamp & 2005 & $\mathrm{~T} 129$ & Santos, J.C. & UFV & 2008 \\
\hline T53 & Pedó, J.C.J. & UFPel & 2007 & $\mathrm{~T} 130$ & Sandini, I.E. & UFPR & 2009 \\
\hline
\end{tabular}


Anexo

Corpus da pesquisa

Conclusão

\begin{tabular}{|c|c|c|c|c|c|c|c|}
\hline No & Doutores & Universidade & Defesa & No & Doutores & Universidade & Defesa \\
\hline T54 & Tedeschi, L.A. & Unisinos & 2007 & $\mathrm{~T} 131$ & Laforga, G. & UFSCar & 2005 \\
\hline T55 & Oliveira, H.M.C. & UFPE & 2008 & $\mathrm{~T} 132$ & Nicoletti, D.R. & UFPel & 2006 \\
\hline T56 & Hernández, C.O. & UFRGS & 2009 & $\mathrm{~T} 133$ & Oliveira, A.R. & Unesp & 2006 \\
\hline T57 & Siliprandi, E.C. & UnB & 2009 & $\mathrm{~T} 134$ & Monteiro, J.M.G. & UFRJ & 2007 \\
\hline T58 & Strapasolas, V.L. & UFSC & 2002 & $\mathrm{~T} 135$ & Rocha, R.N.C. & UFV & 2007 \\
\hline T59 & Weisheimer, N. & UFRGS & 2009 & $\mathrm{~T} 136$ & Silva, G.H. & Unesp & 2008 \\
\hline T60 & Veras, M.M. & UFPE & 2005 & $\mathrm{~T} 137$ & Alves, G.S. & UFPB & 2009 \\
\hline T61 & Siqueira, M.S. & PUC-SP & 2006 & $\mathrm{~T} 138$ & Romero, M.G.C. & Unesp & 2009 \\
\hline T62 & Vasconcelos, C.A. & UFS & 2009 & $\mathrm{~T} 139$ & Gomez, C.U.R. & UFSC & 2006 \\
\hline T63 & Lustosa, M.G. & UFRJ & 2005 & $\mathrm{~T} 140$ & Santos, A.F.S. & UFPR & 2006 \\
\hline T64 & Souza, Z.B. & PUC-SP & 2009 & T141 & Melo Filho, J.B. & UFPE & 2006 \\
\hline T65 & Poletto, Â.R. & UFSC & 2009 & $\mathrm{~T} 142$ & Ribeiro, M.C. & UFV & 2008 \\
\hline T66 & Nascimento, C.A. & Unicamp & 2005 & $\mathrm{~T} 143$ & Martini, L.C.P. & UFRGS & 2000 \\
\hline T67 & Cortez, F.P. & UFPel & 2006 & $\mathrm{~T} 144$ & Silva, G.J. & Unesp & 2009 \\
\hline T68 & Alves, M. & UFSC & 2008 & $\mathrm{~T} 145$ & Araujo, M.E.R. & UFPB & 2008 \\
\hline T69 & Pinto, M.S.V. & Unicamp & 2009 & $\mathrm{~T} 146$ & Spanevello, R.I.M. & UFRGS & 2008 \\
\hline $\mathrm{T} 70$ & Teixeira, V.L. & Unicamp & 2009 & $\mathrm{~T} 147$ & Poliseli, P.C. & UFPR & 2007 \\
\hline $\mathrm{T} 71$ & Sampaio, C.P.S. & Unicamp & 2004 & $\mathrm{~T} 148$ & Bezerra, A.J.A. & UFPel & 2006 \\
\hline $\mathrm{T} 72$ & Franco, J.G.O. & UFPR & 2009 & $\mathrm{~T} 149$ & Róman, R.M.S. & UFV & 2006 \\
\hline $\mathrm{T} 73$ & Hauer, M. & UFPR & 2009 & $\mathrm{~T} 150$ & Oliveira, M.L.F. & Unicamp & 2004 \\
\hline $\mathrm{T} 74$ & Corona, H.M.P. & UFPR & 2006 & $\mathrm{~T} 151$ & Asmus, R.M.F. & UnB & 2004 \\
\hline $\mathrm{T} 75$ & Dias, J.B. & UFPR & 2006 & $\mathrm{~T} 152$ & Zaroni, M.M.H. & Unicamp & 2004 \\
\hline $\mathrm{T} 76$ & Queiroga, J.I.L. & UFPR & 2006 & $\mathrm{~T} 153$ & Pietrafesa, J.P. & UnB & 2002 \\
\hline $\mathrm{T} 77$ & Candiotto, L.Z.P. & UFSC & 2007 & & & & \\
\hline
\end{tabular}

